\title{
Por que os Adolescentes Buscam Fazer Orientação Profissional? Um Estudo Preditivo com Estudantes Brasileiros
}

\author{
Rodolfo Augusto Matteo Ambiel ${ }^{*}, 1$ \\ Orcid.org/0000-0002-3921-8547 \\ Gustavo Henrique Martins ${ }^{1}$ \\ Orcid.org/0000-0002-5125-2553 \\ Débora Noemí Hernández ${ }^{1}$ \\ Orcid.org/0000-0003-1689-3272
}

${ }^{1}$ Universidade São Francisco, Campinas, SP, Brasil

\begin{abstract}
Resumo
Orientadores profissionais que atuam na prática rotineiramente identificam motivos que fazem seus clientes serem mais indecisos ou propensos a buscarem seus serviços, contudo ainda com pouco amparo científico. Sendo assim, o objetivo deste artigo foi verificar o quanto variáveis como personalidade, adaptabilidade de carreira, autoeficácia e exploração vocacional explicam a indecisão vocacional e a intenção de busca por orientação profissional. Participaram deste estudo 237 estudantes dos três anos do ensino médio de uma escola pública, sendo a maior parte do sexo feminino. Foram realizadas duas Análises de Regressão Linear Múltipla, com método forward, sendo usadas como variáveis desfecho a Indecisão e a busca por Orientação Profissional. Os resultados indicaram que os casos foram preditos positivamente por Neuroticismo e Exploração de Si; a Exploração do Ambiente predisse negativamente a Indecisão; a Autoavaliação predisse negativamente a busca por orientação profissional. Conclui-se que os adolescentes buscam orientação profissional por se sentirem vulneráveis emocionalmente, por estarem buscando se conhecer, ou por não acreditarem no seu autoconhecimento profissional.
\end{abstract}

Palavras-chave: Orientação vocacional, estudantes de ensino médio, avaliação psicológica.

\section{Why do Adolescents Seek Career Counseling? A Predictor Study of Brazilian Students}

\begin{abstract}
Career counselors who routinely practice their profession identify the motives that lead their clients to be more indecisive or inclined to seek their services, yet still with little scientific support. That being the case, the present study's aim was to examine the extent to which variables such as personality, career adaptability, self-efficacy and career exploration explain career indecision and the intention to seek

* Endereço para correspondência: Rua Waldemar César da Silveira, 105, Vila Cura D’Ars, Campinas, SP, Brasil, 13045-510. Fone: (19) 3779-3300. E-mail: rodolfo.ambiel@usf.edu.br

Este estudo foi financiado pelo Conselho Nacional de Desenvolvimento (CNPq).
\end{abstract}


Career Counseling. The study enjoyed the participation of 237 sophomores, juniors and seniors from a public high school, and the majority of the students were female. We performed two Multiple Linear Regression Analyses using the forward method, employing Career Indecision and Seeking Career Counseling as the outcome variables. The results indicated that Neuroticism and Self-Exploration positively predicted the cases; Environmental Exploration negatively predicted Career Indecision; and Self-Assessment negatively predicted seeking career counseling. We thus conclude that adolescents seek career counseling either because they feel emotionally vulnerable or because they are seeking selfknowledge, or even because they do not believe in their career-related self-knowledge.

Keywords: Career counseling, high school students, psychological assessment.

\section{Por que los Adolescents Buscan Orientación Prefesional? Un Estudio Predictivo com Estudiantes Brasileños}

\section{Resumen}

Orientadores profesionales que actúan en esta práctica habitualmente identifican motivos que hacen que sus clientes sean más indecisos o propensos a buscar sus servicios, pero sin mucho amparo científico. De esta forma, el objetivo de este artículo fue verificar cual es la influencia cuantitativa de las variables de personalidad, adaptabilidad de carrera, autoeficacia y exploración vocacional en la predicción de la indecisión vocacional y la intención de buscar por orientación profesional. En este estudio participaron 237 estudiantes de los tres años de secundaria de una escuela pública, siendo la mayoría del sexo femenino. Fueron realizadas dos Analices de Regresión Linear Múltiple con el método forward, con las variables desencadenadas la Indecisión y la busca por Orientación Profesional. Los resultados indican que ambos casos fueron previstos positivamente por el Neuroticismo y la Exploración de Sí. Además, Exploración del Ambiente predice negativamente la Indecisión, y de la misma manera la Auto-evaluación predice negativamente la busca por orientación profesional. Concluyendo que los adolescentes buscan la orientación profesional cuando se sienten emocionalmente vulnerables, cuando desean buscar conocerse o por no creer en su propio autoconocimiento profesional.

Palabras clave: Orientación vocacional, estudiantes de secundaria, evaluación psicológica.

A adolescência, que tende a ser estressante e angustiante para os adolescentes por conta de todas as mudanças físicas e sociais típicas, é também o momento do desenvolvimento que abriga a realização das primeiras escolhas por parte dos adolescentes relacionadas a qual profissão o mesmo quer ter no futuro (Reis et al., 2015). Para auxiliar os adolescentes nesta tarefa, os serviços de Orientação Profissional (OP) surgem como uma possível alternativa. No Brasil o termo mais utilizado para fazer referência a tais serviços ainda continua sendo Orientação Profissional (Ribeiro, 2011). Por este motivo OP será o termo utilizado para se referir à orientação neste presente artigo. A OP foi definida por Savickas (1999) como um processo que tem como principal objetivo orientar as pessoas com relação as suas carreiras, a fim de proporcionar a elas uma maior compreensão das características das profissões e um maior autoconhecimento, despertando, assim, potencialidades até o momento desconhecidas.

Em 2004 a Organização para a Cooperação e Desenvolvimento Econômico (OCDE) desenvolveu um guia, denominado de "Orientação Escolar e Profissional: Guia para Decisores", o qual teve como principal objetivo auxiliar gestores no desenvolvimento de políticas públicas eficientes para promoção de orientação. Nesse guia a OCDE conceitualizou a orientação da seguinte forma:

Conjunto de atividades que permitem aos cidadãos de qualquer idade, em qualquer momento das suas vidas, identificar as suas 
aptidões, competências e interesses, tomar decisões importantes a nível escolar, a nível da sua formação e a nível profissional e gerir os seus projetos de vida individuais no estudo, no trabalho e em outros contextos nos quais essas aptidões e competências são adquiridas ou utilizadas. A Orientação é oferecida em diversos contextos: educação, formação, emprego, a nível comunitário e a nível privado. (OCDE, 2004, p. 84)

Vale salientar também que a OP ocupa um importante papel para a sociedade, uma vez que indivíduos com escolhas acertadas se tornam mais satisfeitos, tanto na esfera pessoal quanto profissional, exercendo seus papéis profissionais de forma mais eficiente e produtiva para a sociedade (Carvalho, 1995; Ribeiro \& Uvaldo, 2007). Além disso, o processo OP, por trabalhar questões relativas ao bem-estar individual do orientando, configura-se como "um importante instrumento de promoção de saúde" (Santos, Luna, \& Bardagi, 2014, p. 277). Carvalho (1995) comenta também que o jovem que busca por OP, está à procura de ajuda nas escolhas que tem que realizar para o seu futuro profissional.

Alguns autores mostraram-se interessados em compreender os motivos que fazem com que uma pessoa busque por OP. Ribeiro (2003), por exemplo, realizou um estudo com 252 estudantes do $2^{\circ}$ e $3^{\circ}$ ano do ensino médio de várias escolas públicas da periferia de São Paulo, avaliados por meio de uma entrevista semiaberta. $\mathrm{O}$ autor verificou que a principal demanda que os alunos trouxeram para a OP foi a falta de informação profissional e de fontes de pesquisa relativas à realidade sócio profissional dos mesmos. No mesmo ano, Bardagi, Lassance e Paradiso (2003) publicaram uma pesquisa com uma amostra de 391 estudantes universitários, em meio de curso, de uma universidade federal, os quais responderam a um questionário desenvolvido exclusivamente para esta pesquisa, contendo questões semiestruturadas. As autoras encontraram que a razão que os participantes julgaram ser mais relevante para participar de um processo de OP foi a necessidade de mais informações sobre as profissões, cursos, mercado de trabalho e áreas, fato este que foi interpretado como um possível défi- cit de exploração vocacional no momento de escolha do curso. Por fim, Semensato et al. (2009) realizaram um estudo qualitativo com 20 alunos do $3^{\circ}$ ano do ensino médio, o qual apontou para a existência de três principais motivos que levam esses estudantes a procurar um processo de OP, são estes dúvida, indecisão e falta de informação. Sendo assim, as autoras concluem que os indivíduos procuram OP como forma de auxílio para decidirem sobre suas futuras profissões.

Embora a OP possa ser realizada de diversas formas e com diferentes públicos, a prática com adolescentes que buscam auxílio para escolher o melhor curso universitário para si, ainda é sem dúvidas a mais consolidada (Bonadiman, Scaff, Bardagi, \& Luna, 2016; Melo-Silva, Lassance, \& Soares, 2004). No Brasil o termo OP ficou tradicionalmente relacionado a um processo que visa a ajudar o jovem a refletir melhor nos momentos de indecisão sobre suas escolhas profissionais (Silva, 2010). Como visto, a indecisão vem se mostrando um fator relevante a ser trabalhado no processo de OP, sendo que este já foi indicado como o segundo construto mais estudado na psicologia vocacional, ficando atrás apenas dos interesses profissionais (Kelly \& Lee, 2002). A indecisão vocacional é definida pelas dificuldades encontradas pelos indivíduos no momento que estes precisam tomar decisões relacionadas à carreira (Saka, Gati, \& Kelly, 2008).

Alguns estudos de indecisão com adolescentes no Brasil apontam para a existência de correlações positivas e significativas entre indecisão e ansiedade e depressão, além disso foi visto também que baixos escores de indecisão estão ligados aos adolescentes com escolhas profissionais já definidas. Por outro lado altos escores foram apresentados pelos filhos únicos ou do meio em comparação com os outros (Hutz $\&$ Bardagi, 2006). Outro estudo constatou que a instabilidade de metas e a responsividade combinada dos pais explicaram juntas $12,7 \%$ da indecisão vocacional, sendo a instabilidade de metas uma variável mediadora para essa relação (Magalhães, Alvarenga, \& Teixeira, 2012). A indecisão mostrou-se correlacionada negativamente e com magnitude fraca com otimismo (Campos \& Noronha, 2015). Por fim, foi visto que as pessoas 
que apresentaram mais indecisão apresentaram maiores escores em autoeficácia para escolha profissional e exploração de si (Ambiel \& Hernández, 2016).

Muitas vezes o principal foco em um processo de OP é o de auxiliar o orientando na identificação das suas características a fim de minimizar a sua indecisão (Campos \& Noronha, 2015; Noronha \& Ambiel, 2008). Sendo assim, uma possível estratégia comportamental para sanar essa dificuldade é a de exploração (Cunha \& Faria, 2009), uma vez que a indecisão pode ser considerada um resultado de uma exploração inefetiva (Faria, Taveira, \& Saavedra, 2008). A exploração vocacional é mais comum na adolescência e início da idade adulta, sendo definida como comportamentos voltados para a solução de problemas, descobertas, experimentação e conhecimento sobre si e sobre o mundo de trabalho, sendo esta fundamental para a escolha profissional (Brown \& Lent, 2005; Joordan, 1963).

Além da indecisão e da exploração vocacionais, alguns pesquisadores estão interessados também em estudar o funcionamento auto regulatório dos indivíduos e como este está envolvido nos processos de decisão de carreira. Neste sentido, dois construtos são amplamente estudados na literatura nacional e internacional, a autoeficácia e a adaptabilidade de carreira. Embora pertençam a teorias diferentes, a saber teoria sociocognitiva (Lent, Brown, \& Hackett, 1994) e teoria da construção de carreira (Savickas, 2005), respectivamente, ambas mediam as relações entre disposições e comportamentos e por isso são designadas como processos auto regulatórios (Lent et al., 1994; Rossier, 2015). Por definição a autoeficácia é entendida como as crenças do indivíduo em sua própria capacidade de organizar e executar cursos de ações necessários para alcançar certas realizações (Bandura, 1997). Por outro lado, a adaptabilidade de carreira é definida como sendo um "construto psicossocial que denota a prontidão e os recursos de um indivíduo para lidar com as tarefas do desenvolvimento profissional, atuais e iminentes, transições ocupacionais e traumas pessoais" (Savickas, 2005, p. 51). Um estudo feito por Guan et al. (2016) com 731 estudantes universitários chineses, constatou a correlação positiva e significativa entre a autoeficácia para tomada de decisão de carreira e a adaptabilidade de carreira.

Alguns orientadores buscam também compreender as relações que os aspectos psicológicos e disposicionais têm com o comportamento vocacional, ou seja como as características psicológicas presentes nos próprios indivíduos se relacionam com os construtos relacionados à carreira. Dentre os construtos psicológicos mais estudados neste âmbito encontra-se a personalidade, a qual é mundialmente compreendida e pesquisada por meio do modelo Big Five. Neste sentido há diversos estudos no Brasil que buscaram relacionar a personalidade com outros construtos da psicologia vocacional como: interesses profissionais (Bueno, Lemos, \& Tomé, 2004; Noronha, Mansão, \& Nunes, 2012; Primi et al., 2002; Valentini, Teodoro, \& Balbinotti, 2009), autoeficácia profissional, planejamento de carreira, decisão de carreira e exploração (Ourique \& Teixeira, 2012), autoeficácia para escolha profissional (Ambiel \& Noronha, 2016), adaptabilidade de carreira (Bardagi \& Albanaes, 2015; Teixeira, Bardagi, Lassance, Magalhães, \& Duarte, 2012), carreira proteana (Borges \& Andrade, 2014), entre outros.

Como visto até aqui, muitos fatores estão envolvidos no comportamento vocacional, mas de fato quais deles podem estar ligados ao desejo de uma pessoa buscar por orientação profissional? Visando responder a esta pergunta, alguns autores reportaram em seus estudos para motivos externos ao indivíduo (Bardagi et al., 2003; Ribeiro, 2003; Semensato et al., 2009), não sendo encontradas pesquisas que tenham investigado a influência de motivos internos do indivíduo para a sua intenção em buscar o serviço de orientação profissional. Portanto o objetivo deste estudo foi verificar o quanto variáveis como personalidade, adaptabilidade, autoeficácia e exploração vocacional explicam a indecisão vocacional e a intenção de busca por orientação profissional. Uma vez que ao se conhecer os fatores psicológicos envolvidos nestes processos (indecisão e busca por orientação profissional), os orientadores poderão trabalhar também a nível preventivo a fim de oferecer auxílio para tais demandas. 
Com base no levantamento feito da literatura científica da área, hipotetiza-se com este estudo: H1) que a indecisão será explicada positivamente por abertura e neuroticismo e negativamente por conscienciosidade e autoeficácia (Ambiel \& Hernández, 2016; Campos \& Noronha, 2015; Hutz \& Bardagi, 2006; Ourique \& Teixeira, 2012); H2) a exploração, tanto de si, quanto do ambiente será um construto preditor positivo da indecisão vocacional e da busca por OP (Ambiel \& Hernández, 2016; Bardagi et al., 2003; Faria et al., 2008; Ribeiro, 2003; Semensato et al., 2009).

\section{Método}

\section{Participantes}

A amostra (selecionada por conveniência) deste estudo foi composta por 237 estudantes do ensino médio pertencentes a uma escola pública do interior do estado de São Paulo. A média de idade dos adolescentes foi de 16,31 ( $D P=0,98)$, com idades variando de 14 a 19 anos, e o sexo predominante foi o feminino $(53,2 \%)$, sendo que duas pessoas não concederam essas informações. A respeito da série $39,7 \%$ eram do primeiro ano, $28,7 \%$ do segundo ano e $30,8 \%$ do terceiro ano do ensino médio, do total três pessoas não informaram sua série.

\section{Instrumentos}

Questionário de identificação. Contém perguntas sobre idade, sexo e série, além de uma pergunta fechada sobre a intenção dos adolescentes em buscar por OP. Antes da pergunta foi feita uma breve explicação sobre o que é o processo de Orientação Profissional e após a explicação o participante deveria responder ao seguinte enunciado: "Considerando a maneira com que você está pensando hoje sobre o seu futuro", marcando umas das três opções a seguir: (a) Eu não procuraria uma orientação profissional, pois já tenho certeza do que quero fazer no futuro; (b) Embora eu já saiba o que quero fazer no futuro, eu faria uma orientação profissionais, pois poderia me ajuda a ter certeza se estou no caminho certo; (c) Eu procuraria uma orientação profissional, pois não tenho ideia do que vou fazer no ano que vem. Para fins de análise, os dados foram tabulados como 1 (opção a), 2 (opção b) e 3 (opção c), sendo que 1 significava uma menor intenção do estudante em buscar por OP e 3 significava uma maior intenção do estudante em buscar por OP.

Marcadores Reduzidos para a Avaliação da Personalidade (Hauck, Machado, Teixeira, \& Bandeira, 2012; Hauck, Teixeira, Machado, \& Bandeira, 2012). Avalia a personalidade por meio de adjetivos baseados no modelo dos Cinco Grandes Fatores. É composto por 25 marcadores (e.g. Comunicativa), divididos igualmente em cinco dimensões: extroversão, socialização, conscienciosidade, neuroticismo e abertura. A chave de resposta é dada em uma escala Likert de cinco pontos, sendo (1) "discordo totalmente" e (5) "concordo totalmente". O instrumento apresenta índices satisfatórios de precisão, com Alfa de Cronbach variando de 0,61 (abertura) a 0,83 (extroversão). No estudo feito por Hauck, Teixeira, et al. (2012) com amostras de estudantes do ensino médio, foram confirmadas as boas propriedades psicométricas do instrumento para esta população. No presente estudo foram constatados os seguintes Alfas de Cronbach: 0,71 (socialização), 0,70 (conscienciosidade), 0,66 (neuroticismo), 0,54 (abertura) e 0,46 (extroversão).

Escala de Adaptabilidade de Carreira CAAS (Teixeira et al., 2012). Avalia os fatores da adaptabilidade de carreira e deve ser respondida mediante o quanto a pessoa acredita ter desenvolvido cada habilidade, numa escala Likert que varia de "muito pouco" (1) a "plenamente" (5). A escala é composta por 24 itens, que por meio de análise fatorial confirmatória agruparam-se em quatro fatores, tal qual hipotetizado, quais sejam, preocupação, controle, curiosidade e confiança. Estes fatores obtiveram valores satisfatórios de precisão com Alfa de Cronbach variando de 0,76 (controle) a 0,82 (confiança) e 0,91 para o escore geral. Foi realizado um estudo com adolescentes, o qual confirmou as propriedades psicométricas da CAAS para essa população, apresentando Alfas de Cronbach variando de 0,81 (controle e curiosidade) a 0,86 (confiança; Ambiel, Carvalho, Martins, \& Tofoli, 2016). 
Escala de Autoeficácia para Escolha Profissional - EAE-EP (Ambiel \& Noronha, 2014). Nesta escala o participante deve responder à questão "quanto você acredita em sua capacidade de . ..", numa escala do tipo Likert de quatro pontos, sendo que 1 representa "acredito pouco" e 4 "Acredito muito". É composta por 48 itens que se dividem em quatro fatores, sendo estes autoavaliação (crença do indivíduo sobre suas capacidades se descrever e decidir-se por uma profissão), coleta de informação ocupacional (crença do indivíduo em ser capaz de buscar informações sobre profissões se valendo de diversas estratégias), busca de informações profissionais práticas (crença do sujeito na sua capacidade para obter informações sobre o mundo do trabalho por meio de relacionamentos interpessoais) e planejamento de futuro (crença em poder contemplar questões referentes a sua futura formação, profissão ou questões financeiras). O Alfa de Cronbach deste instrumento variou de 0,79 (planejamento de futuro) a 0,88 (autoavaliação), e 0,94 para a escala total. No presente estudo os Alfas de Cronbach encontrados foram todos acima de 0,77.

Escalas de Exploração Vocacional EEV (Teixeira, Bardagi, \& Hutz, 2007; Teixeira \& Dias, 2011). Avaliam o comportamento exploratório dos estudantes do ensino médio, por meio de dois fatores, sendo eles exploração de si (busca por maior autoconhecimento) e exploração do ambiente (busca por alternativas educacionais e profissionais). É composta por 24 itens, igualmente divididos entre os fatores, e avaliados por meio de uma escala Likert de cinco pontos variando de "raramente ou nunca" (1) a "muito frequentemente ou sempre" (5). A consistência interna da escala foi considerada favorável, com Alfa de Cronbach de 0,81 para Exploração do Ambiente a 0,83 para Exploração de Si. Da mesma forma, no presente estudo os Alfas de Cronbach encontrados foram 0,85 (exploração do ambiente) e 0,82 (exploração de si).

Escala de Indecisão Vocacional - EIV (Teixeira \& Magalhães, 2001). A escala busca medir o nível de indecisão vocacional de estudantes do ensino médio em um modelo unidimensional. É composta por 30 itens avaliados em uma escala Likert de cinco pontos, variando de "a frase é totalmente falsa a meu respeito" (1) a "a frase é totalmente verdadeira a meu respeito" (5). A precisão da escala foi considerada satisfatória com Alfa de Conbrach de 0,92 (indecisão vocacional). No presente estudo a precisão da escala se manteve, com Alfa de Cronbach de 0,88.

\section{Procedimentos}

Procedimentos éticos. Inicialmente foi realizado o primeiro contato com a coordenadora do ensino médio da escola e, após a explicação do projeto, ela concedeu autorização para a realização da pesquisa. O projeto foi submetido e aprovado pelo Comitê de Ética em Pesquisa da Universidade São Francisco (número do parecer: 810.064). Após o parecer, a escola foi contatada novamente para a entrega dos Termos de Consentimento Livre Esclarecido (TCLE), os quais foram encaminhados aos pais dos participantes, sendo informado que somente participariam da pesquisa aqueles cujos responsáveis legais consentirem. A própria coordenadora ficou responsável por receber de volta os TCLEs preenchidos e assinados e, assim que a maioria dos alunos já haviam entregue (cerca de $90 \%$ daqueles que haviam sido convidados), ela avisou os pesquisadores, que agendaram a data da aplicação. Como contrapartida, cerca de um mês após a coleta, um dos autores retornou à escola e fez uma palestra sobre escolha profissional para todos os alunos das salas participantes, independente de terem ou não participado da pesquisa.

Coleta de dados. A coleta foi feita de maneira coletiva em sala de aula, em período letivo, com aproximadamente 40 alunos em cada sala, com duração aproximada de 40 minutos. Os instrumentos foram aplicados por dois psicólogos e dois estudantes de psicologia bolsistas de iniciação científica.

Análise de dados. Os dados foram tabulados e analisados em planilhas do SPSS, sendo que após essa etapa foram realizadas análises 
descritivas da amostra e das variáveis utilizadas para este estudo. Foi analisada também a precisão por consistência interna dos fatores por meio do Alfa de Cronbach. Em relação aos valores de Alfa, foram tomadas como referência as indicações de George e Mallery (2002) que defendem que valores de Alfa menores que 0,50 são considerados inaceitáveis, Alfas entre 0,50 e 0,59 são ruins, Alfas entre 0,60 e 0,69 são questionáveis, Alfas entre 0,70 e 0,79 são aceitáveis, Alfas entre 0,80 a 0,89 são bons e Alfas acima de 0,90 são excelentes. Além disso, foram comparados os tamanhos de efeitos das médias das variáveis utilizadas entre os três anos do ensino médio. Para interpretação destes resultados, foram utilizadas as considerações feitas por Cohen (1988), o qual aponta que valores de $d=0,2$ são considerados tamanhos de efeito pequenos, $d=0,5$ são considerados médios e $d=0,8$ são considerados grandes.

Após a análise de consistência interna dos instrumentos, foram realizadas duas Análises de Regressão Linear Múltipla, com método forward. Todos os pressupostos para a realização da análise de regressão foram realizados e indicaram a possibilidade da análise ser feita. De acordo com os pressupostos de Green (1991), o tamanho amostral deste estudo encontra-se adequado para a realização da Análise de Regressão Linear. Na primeira análise foi adotada como variável de desfecho a indecisão vocacional e a

s variáveis independentes foram personalidade (primeiro bloco), adaptabilidade de carreira e autoeficácia para escolha profissional (segundo bloco) e exploração vocacional (terceiro bloco). Na sequência foi realizada a segunda Análise de Regressão, também com método forward, sendo que nesta análise a variável de desfecho foi a busca por orientação profissional, mantendo a mesma sequência de variáveis independentes nos blocos. As variáveis foram alocadas desta forma nos seus respectivos blocos tendo em vista um modelo que se inicia nos aspectos disposicionais (primeiro bloco), continua para os processos auto regulatórios (segundo bloco) e termina nos aspectos comportamentais (terceiro bloco), seguindo o modelo de desenvolvimento vocacional de Lent et al. (1994).

\section{Resultados}

A Tabela 1 apresenta a média e o desvio padrão dos participantes da amostra geral e separada por série, em relação às variáveis utilizadas neste estudo. A respeito das amplitudes das médias, vale ressaltar que esta variou de um a cinco em todas as variáveis, exceto nos fatores da autoeficácia e da variável busca por orientação profissional, as quais variaram de um a quatro e de um a três, respectivamente.

Os tamanhos de efeito das médias entre as três séries do ensino médio foram todos pequenos ou nulos, indicando, de forma geral, que não houve em nenhuma série uma prevalência nos escores das variáveis avaliadas, em comparação com as outras séries. As variáveis que apresentaram tamanhos de efeito pequenos entre as séries foram conscienciosidade, busca de informações ocupacionais práticas, exploração do ambiente, exploração de si, indecisão vocacional e busca por orientação profissional. A seguir a Tabela 2 apresenta a Análise de Regressão com as variáveis preditoras em relação à indecisão vocacional.

A partir da Tabela 2 foi possível verificar que apesar de serem incluídas diversas variáveis na análise, com intuito de explicar a indecisão vocacional, o melhor modelo explicativo foi formado apenas pelos fatores neuroticismo e exploração de si, sendo que estes dois fatores explicaram $9 \%$ da indecisão vocacional dos adolescentes. A respeito do sentido da predição, foi visto que tanto o neuroticismo como a exploração de si predizem positivamente a indecisão, ou seja, quanto maior o nível de neuroticismo e exploração de si do adolescente, mais indeciso ele se avalia em relação à sua carreira. Por fim, na Tabela 3 serão descritos os resultados da Análise de Regressão, a fim de explicar a busca por orientação profissional. 
Tabela 1

Descritiva da Amostra Separada por Série e Tamanhos de Efeito

\begin{tabular}{|c|c|c|c|c|c|c|c|}
\hline Variáveis & $M(D P)$ Total & $\begin{array}{c}M(D P) \\
1^{\circ} \text { ano }\end{array}$ & $\begin{array}{c}M(D P) \\
2^{\circ} \text { ano }\end{array}$ & $\begin{array}{c}M(D P) \\
3^{\circ} \text { ano }\end{array}$ & $d 1^{\circ} \times 2^{\circ}$ & $d 1^{\circ} \times 3^{\circ}$ & $d 2^{\circ} \times 3^{\circ}$ \\
\hline Extroversão & $3,05(0,49)$ & $3,08(0,46)$ & $3,02(0,46)$ & $3,04(0,55)$ & 0,13 & 0,08 & 0,04 \\
\hline Socialização & $3,87(0,55)$ & $3,86(0,52)$ & $3,91(0,54)$ & $3,81(0,60)$ & 0,09 & 0,09 & 0,17 \\
\hline Conscienciosi. & $3,83(0,59)$ & $3,91(0,57)$ & $3,81(0,62)$ & $3,74(0,60)$ & 0,17 & $0,29^{*}$ & 0,11 \\
\hline Neuroticismo & $2,71(0,72)$ & $2,71(0,84)$ & $2,67(0,65)$ & $2,77(0,65)$ & 0,05 & 0,08 & 0,15 \\
\hline Abertura & $3,16(0,63)$ & $3,17(0,57)$ & $3,20(0,61)$ & $3,12(0,70)$ & 0,05 & 0,08 & 0,12 \\
\hline Preocupação & $3,76(0,82)$ & $3,73(0,87)$ & $3,76(0,78)$ & $3,79(0,83)$ & 0,04 & 0,07 & 0,04 \\
\hline Controle & $3,84(0,81)$ & $3,78(0,84)$ & $3,87(0,85)$ & $3,89(0,75)$ & 0,11 & 0,14 & 0,03 \\
\hline Curiosidade & $3,50(0,82)$ & $3,53(0,78)$ & $3,46(0,90)$ & $3,53(0,79)$ & 0,08 & 0,00 & 0,08 \\
\hline Confiança & $3,91(0,79)$ & $3,90(0,79)$ & $3,95(0,75)$ & $3,89(0,83)$ & 0,06 & 0,01 & 0,08 \\
\hline Autoavaliação & $3,09(0,47)$ & $3,13(0,45)$ & $3,09(0,45)$ & $3,04(0,51)$ & 0,09 & 0,19 & 0,10 \\
\hline Coleta Info. & $2,99(0,60)$ & $3,02(0,62)$ & $2,95(0,54)$ & $2,97(0,64)$ & 0,12 & 0,08 & 0,03 \\
\hline Busca Info. & $2,89(0,59)$ & $2,98(0,56)$ & $2,83(0,56)$ & $2,81(0,65)$ & $0,27^{*}$ & $0,28^{*}$ & 0,03 \\
\hline Planejamento & $3,20(0,54)$ & $3,20(0,58)$ & $3,23(0,44)$ & $3,15(0,56)$ & 0,06 & 0,09 & 0,16 \\
\hline Exploração A. & $3,26(0,80)$ & $3,36(0,84)$ & $3,28(0,76)$ & $3,13(0,78)$ & 0,10 & $0,28^{*}$ & 0,19 \\
\hline Exploração Si & $3,48(0,73)$ & $3,58(0,72)$ & $3,51(0,72)$ & $3,34(0,74)$ & 0,10 & $0,33^{*}$ & $0,23^{*}$ \\
\hline Indecisão & $2,61(0,66)$ & $2,71(0,65)$ & $2,56(0,65)$ & $2,50(0,67)$ & $0,23^{*}$ & $0,32^{*}$ & 0,09 \\
\hline Busca por OP & $2,01(0,59)$ & $1,98(0,59)$ & $2,09(0,48)$ & $1,97(0,67)$ & $0,20^{*}$ & 0,02 & $0,20^{*}$ \\
\hline
\end{tabular}

Nota. $1^{\circ}$ ano $N=93 ; 2^{\circ}$ ano $N=68 ; 3^{\circ}$ ano $N=73 ; M$ : média; $D P$ : desvio padrão; $d$ : tamanho do efeito; ${ }^{*}$ : tamanho de efeito pequeno.

Tabela 2

Regressão entre as Variáveis em Relação à Indecisão Vocacional

\begin{tabular}{lccc}
\hline Variáveis preditoras & \multicolumn{3}{c}{ Indecisão Vocacional } \\
\cline { 2 - 4 } & $\beta$ & $p$ & $\mathrm{IC} 95 \%(\beta)$ \\
\hline Neuroticismo & 0,21 & $<0,01$ & 0,$08 ; 0,30$ \\
Exploração de Si & 0,23 & $<0,01$ & 0,$10 ; 0,32$ \\
& & $R^{2}=0,09$ & \\
\hline
\end{tabular}

Nota. $R^{2}$ : coeficiente de determinação ajustado; $\beta$ : coeficiente de regressão linear ajustado; IC: intervalo de confiança. Não foram informados os valores das variáveis excluídas do modelo. 
com Estudantes Brasileiros.

Tabela 3

Regressão entre as Variáveis em Relação à Busca por Orientação Profissional

\begin{tabular}{lccc}
\hline Variáveis preditoras & \multicolumn{3}{c}{ Busca por Orientação Profissional } \\
\cline { 2 - 4 } & $\beta$ & $p$ & $\mathrm{IC} 95 \%(\beta)$ \\
\hline Neuroticismo & 0,22 & $<0,01$ & 0,$08 ; 0,27$ \\
Autoavaliação & $-0,33$ & $<0,01$ & $-0,59 ;-2,23$ \\
Exploração de Si & 0,17 & 0,02 & 0,$02 ; 0,25$ \\
& & $R^{2}=0,14$ & \\
\hline
\end{tabular}

Nota. $R^{2}$ : coeficiente de determinação ajustado; $\beta$ : coeficiente de regressão linear ajustado; IC: intervalo de confiança. Não foram informados os valores das variáveis excluídas do modelo.

Assim como na análise anterior (Tabela 2), foram inseridas como variáveis independentes todos os fatores de personalidade, adaptabilidade de carreira, autoeficácia para escolha profissional e exploração vocacional. Destas variáveis, apenas o neuroticismo, a autoavaliação e a exploração de si, foram preditoras no melhor modelo explicativo, o qual apresentou um nível de explicação de $14 \%$ da busca por orientação profissional. Sendo assim, os fatores que explicaram positivamente a busca por orientação profissional foram neuroticismo e exploração de si, sendo estes os mesmos fatores que predisseram a indecisão vocacional. Por outro lado, o fator que apresentou o maior nível explicativo para a busca de orientação profissional foi a autoavaliação, predizendo negativamente a variável dependente.

\section{Discussão}

O presente estudo verificou o quanto a personalidade, a adaptabilidade de carreira, a autoeficácia e a exploração vocacional explicam a indecisão vocacional e a intenção de busca por orientação profissional. A partir dos resultados podem-se corroborar parcialmente as hipóteses levantadas neste estudo. A H1 previa que a indecisão seria explicada positivamente por abertura e neuroticismo e negativamente por conscienciosidade e autoeficácia. Entretanto, os resultados indicaram que apenas o neuroticismo foi capaz de predizer a indecisão neste estudo. O neuro- ticismo refere-se às tendências do indivíduo a experimentar emoções negativas, como tristeza e ansiedade. Por esse motivo ter altas pontuações neste fator refere-se a uma maior instabilidade emocional (John, Naumann, \& Soto, 2008). Sendo assim, este dado sugere que os adolescentes que vivenciam uma maior instabilidade emocional são mais indecisos em relação a sua profissão em comparação àqueles menos ansiosos e deprimidos, ou com mais afetos positivos. A literatura apontou ainda que a indecisão se correlacionou positivamente com ansiedade e depressão (Hutz \& Bardagi, 2006) e com pessimismo (Campos \& Noronha, 2015), sendo estas características presentes em pessoas com neuroticismo elevado. Além destes estudos, Ourique e Teixeira (2012) também constataram que o neuroticismo é um fator preditor negativo de decisão de carreira de universitários, sugerindo que os resultados aqui encontrados não sejam efeito da idade ou experiência acadêmica.

Além do neuroticismo, a exploração de si também foi preditora de indecisão profissional, assim como hipotetizado a partir de outros estudos (Ambiel \& Hernández, 2016; Faria et al., 2008). A exploração de si foi a variável que melhor explicou a indecisão vocacional, predizendo positivamente a indecisão. Neste caso, os resultados mostraram que os adolescentes que mais buscam se conhecer são aqueles que apresentam mais indecisão vocacional. Isto pode ser explicado pelo fato de que à medida que o indivíduo se questiona a respeito dos seus interesses e valores 
(implicando em seu autoconhecimento) ele pode também despertar dúvidas a respeito de si mesmo, vivenciando assim, dificuldades para tomar decisões sobre a sua carreira.

Ainda nesta direção, $\mathrm{H} 2$ foi parcialmente corroborada, uma vez que esta previa que a exploração de si e exploração do ambiente seriam preditoras de indecisão vocacional e de busca por orientação profissional. Portanto, a partir desta hipótese, foi visto também que a exploração de si foi uma variável preditora da busca por orientação profissional. Nesse caso, esses resultados indicaram que os estudantes que olham mais para dentro de si mesmos, visando se compreenderem melhor, são aqueles que apresentam mais intenção por buscar o serviço de orientação profissional. Este resultado corrobora os pressupostos teóricos de que um dos objetivos do trabalho de OP é promover no orientando maior autoconhecimento profissional. Possivelmente por este motivo, os adolescentes que buscavam se conhecer melhor acabavam suscitando dúvidas a respeito de si, o que geravam neles maiores níveis de indecisão, e assim consequentemente demonstravam ter mais intenção de buscar o serviço de OP, uma vez que encontram nesse serviço uma forma de auxílio para o processo de autoconhecimento (Santos et al., 2014).

Outro fator que predisse positivamente a busca por orientação profissional foi o neuroticismo, indicando que os adolescentes mais instáveis emocionalmente são aqueles que consideram precisar mais deste tipo de serviço, provavelmente pelo fato de estes adolescentes necessitarem naquele momento, de mais ajuda em suas vidas e, consequentemente, verem neste serviço uma possível oportunidade de receber ajuda. Pesquisas apontam que pessoas com um maior nível de neuroticismo tendem a procurar mais ajuda para seus problemas de saúde mental (Parslow \& Jorm, 2000; Zoonen et al., 2015).

Por último, o fator que melhor explicou a busca por orientação profissional, foi a Autoavaliação, sendo este um preditor negativo. Sobre este resultado, é possível entender que os adolescentes que acreditam se conhecer profissionalmente bem são aqueles que indicaram não ter intenção em buscar por orientação profissional.
Isto se explica pelo fato de que quanto mais o adolescente tem uma crença sobre a sua capacidade de autoconhecimento profissional (Ambiel \& Noronha, 2014), mais enraizada fica a ideia de ele não precisar de ajuda em relação a sua carreira. Outro ponto que deve ser considerado é o fato de que alguns adolescentes não reconhecem a necessidade de buscar por orientação profissional, devido a um nível de autoconhecimento disfuncional influenciado pelo excesso de confiança (Moores \& Chang, 2009).

Em outros estudos que buscaram identificar as demandas trazidas pelos orientandos que participavam de processos de orientação profissional, foram encontradas como principais demandas: a falta de informação profissional e de fontes de pesquisa (Ribeiro, 2003), necessidade de mais informações profissionais e educacionais (Bardagi et al., 2003), dúvida, indecisão e falta de informação (Semensato et al., 2009). Nesses estudos, os autores por meio de estudos exploratórios e/ou qualitativos constataram que as principais demandas se referiam a variáveis externas ao indivíduo (e.g. falta de informação). Contudo este dado foi diferente do encontrado no presente estudo, uma vez que as variáveis preditoras de busca por orientação profissional encontradas são internas do indivíduo (exploração de si, neuroticismo e autoavaliação). Sendo assim, é possível deduzir que quando os alunos responderam abertamente sobre os motivos que os levaram a buscar por orientação profissional, os mesmos reportaram motivos externos (Bardagi et al., 2003; Ribeiro, 2003; Semensato et al., 2009). Em contraste, na presente pesquisa, os adolescentes tiveram menos controle sobre as variáveis, por serem avaliadas por meio de escalas psicométricas, o que permitiu identificar algumas motivações internas para a busca de um serviço de orientação profissional.

Por fim, foi observado que as duas variáveis preditoras de indecisão vocacional foram igualmente preditoras de busca por orientação profissional, o que indica que há pontos comuns entre estas duas variáveis. A única variável que diferenciou o modelo explicativo da indecisão vocacional do modelo explicativo de busca por orientação profissional foi a autoavaliação, pre- 
sente somente no último modelo. Neste sentido, quanto maior os níveis de neuroticismo e exploração de si do adolescente, mais indecisos eles se apresentam e acabam por enxergar na orientação profissional uma possível ajuda para a sua instabilidade emocional e/ou dúvidas em relação a carreira. Entretanto, a crença do indivíduo na sua capacidade de se conhecer bem não impactou em sua indecisão, mas sim na baixa intenção do adolescente buscar orientação profissional.

A partir deste artigo é possível ampliar o conhecimento a respeito de algumas variáveis que estão envolvidas na explicação da indecisão vocacional e da intenção de busca de adolescentes por orientação profissional. Contudo, torna-se importante considerar algumas limitações deste estudo, especificamente no que se refere à precisão dos fatores de personalidade, sendo que dois fatores (extroversão e abertura) apresentaram Alfa de Cronbach insatisfatórios. Da mesma forma, os estudos de construção dos Marcadores Reduzidos para a Avaliação da Personalidade também apontaram para Alfas parecidos (Hauck, Machado, et al., 2012; Hauck, Teixeira, et al., 2012), o que indica não ser uma característica específica da amostra deste estudo. Por essa razão, recomenda-se que em pesquisas futuras seja replicado o presente estudo, utilizando outras medidas de personalidade a fim de testar com maior confiabilidade, os impactos desta variável nas variáveis de desfecho aqui testadas.

Outro ponto que deve ser melhor delimitado diz respeito a variável busca por orientação profissional, a qual mediu a intenção que o adolescente tem de buscar orientação profissional e não se o adolescente procurou a orientação profissional de fato. Sendo assim, é relevante que pesquisas futuras, verifiquem se as variáveis que predizem intenção de busca por orientação profissional são as mesmas que predizem a ação de buscar o serviço de orientação profissional. Além disso, outra hipótese que deve ser testada refere-se ao processo de orientação profissional ser uma possível fonte de indecisão uma vez que este visa promover a exploração por parte do orientando.

Embora os resultados encontrados sejam relevantes para o entendimento destes construtos, o tamanho da amostra e o baixo coeficiente de regressão encontrado apontam para uma limitação do presente estudo. Sendo assim, ainda são necessárias novas pesquisas que realizem coleta de dados em escolas particulares e com amostras mais representativas e pesquisas que visem a explicar as mesmas variáveis investigadas no presente estudo, utilizando outras ainda não testadas, como características sociodemográficas e escolares.

De fato, com este estudo é possível compreender de forma mais ampla as variáveis envolvidas em questões tão importantes para os orientadores profissionais, quais sejam a indecisão vocacional e a intenção de um adolescente buscar por orientação profissional. Em síntese, os resultados do presente estudo sugerem que os adolescentes buscam orientação profissional porque sentem-se vulneráveis emocionalmente ou por estarem buscando autoconhecimento, ou ainda por terem dúvidas acerca do nível ou da quantidade do seu autoconhecimento profissional. Sendo assim, cabe aos orientadores, educadores e familiares desenvolverem formas de minimizar ou promover tais características, especificamente autoeficácia para autoavaliação e exploração de si, a fim de colaborar para uma escolha profissional mais acertada por parte destes adolescentes (Silva \& Marques, 2015). Tais características poderiam ser promovidas através da disponibilização de meios para que os adolescentes tenham contato com informações sobre seus interesses, habilidades, valores e necessidades.

\section{Referências}

Ambiel, R. A. M., Carvalho, L. F., Martins, G. H., \& Tofoli, L. (2016). Comparing the adaptabilities of Brazilian adolescent students and adult workers. Journal of Vocational Behavior, 94, 20-27. doi: 10.1016/j.jvb.2016.02.005

Ambiel, R. A. M., \& Hernández, D. N. (2016). Relações entre autoeficácia para escolha profissional, exploração e indecisão vocacional. Revista Brasielira de Orientação Profissional, 17(1), 67-75. Recuperado em http://pepsic.bvsalud. org/scielo.php?script=sci_issues\&pid=1679$-3390 \& \operatorname{lng}=\mathrm{pt \&}$ rm $=$ iso 
Ambiel, R. A. M., \& Noronha, A. P. P. (2014). Escala de Autoeficácia para Escolha Profissional (EAE-EP): Manual técnico (2. ed.). São Paulo, SP: Casa do Psicólogo.

Ambiel, R. A. M., \& Noronha, A. P. P. (2016). Professional choice self-efficacy: Predicting traits and personality profiles in high school students. Psicologia: Reflexão e Crítica, 29. doi: 10.1186/ s41155-016-0021-0

Bandura, A. (1997). Self-efficacy: The exercise of control. New York: Freeman.

Bardagi, M. P., \& Albanaes, P. (2015). Relações entre adaptabilidade de carreira e personalidade: Um estudo com universitários ingressantes brasileiros. Revista PSICOLOGIA: Revista da Associação Portuguesa de Psicologia, 29(1), 35-44. Recuperado em http://www.scielo.mec. pt/pdf/psi/v29n1/v29n1a04.pdf

Bardagi, M. P., Lassance, M. C. P., \& Paradiso, Â. C. (2003). Trajetória acadêmica e satisfação com a escolha profissional de universitários em meio de curso. Revista Brasileira de Orientação Profissional, 4(1/2), 153-166. Recuperado em http:// pepsic.bvsalud.org/pdf/rbop/v4n1-2/v4n1-2a13. pdf

Bonadiman, M. D., Scaff, L. A., Bardagi, M. P., \& Luna, I. N. (2016). Perfil dos usuários do LIOP - Laboratório de Informação e Orientação Profissional da UFSC: Mudanças observadas nos últimos anos. Caminho Aberto, 1(2), 91-100. Recuperado em http://periodicos.ifsc.edu.br/index.php/caminhoaberto/article/view/1880

Borges, L. F. L., \& Andrade, A. L. (2014). Preditores da carreira proteana: Um estudo com universitários. Revista Brasileira de Orientacao Profissional, 15(2), 153-163. Recuperado em http:// pepsic.bvsalud.org/pdf/rbop/v15n2/06.pdf

Brown, S. D., \& Lent, R. W. (2005). Career development and counseling: Putting theory and research to work. Hoboken, NJ: John Wiley \& Sons.

Bueno, J. M. H., Lemos, C. G., \& Tomé, F. A. M. F. (2004). Interesses profissionais de um grupo de estudantes de psicologia e suas relações com inteligência e personalidade. Psicologia em Estudo, 9(2), 271-278. doi: 10.1590/S141373722004000200013

Campos, R. R. F., \& Noronha, A. P. P. (2015). A relação entre indecisão profissional e otimismo disposicional em adolescentes. Temas em Psicolo- gia, 24(1), 219-232. doi: 10.9788/TP2016.1-15

Carvalho, M. M. M. J. (1995). Orientação profissional em grupo: Teoria e técnica. São Paulo, SP: Editorial Psy.

Cohen, J. (1988). Statistical power analysis for the behavioral sciences ( $2^{\text {nd }}$ Ed.). Hillsdale, NJ: Lawrence Earlbaum Associates.

Cunha, M. C. T. C. S. B., \& Faria, L. C. (2009). Efeito da intervenção psicológica vocacional na indecisão e comportamento exploratório. Psicologia Ciência e Profissão, 29(3), 558573. Recuperado em http://www.redalyc.org/ $\mathrm{html} / 2820 / 282021777010 /$

Faria, L. C., Taveira, M. C., \& Saavedra, L. M. (2008). Exploração e decisão de carreira numa transição escolar: Diferenças individuais. Revista Brasileira de Orientação Profissional, 9(2), 17-30. Recuperado em http://pepsic.bvsalud. org/pdf/rbop/v9n2/v9n2a04.pdf

George, D., \& Mallery, P. (2002). SPSS for Windows step by step: A simple guide and reference. 11.0 update ( $4^{\text {th }}$ Ed.). Boston, MA: Allyn \& Bacon.

Green, S. B. (1991). How many subjects does it take to do a regression analysis? Multivariate Behavioral Research, 26(3), 449-510. doi: 10.1207/ s15327906mbr2603_7

Guan, M., Capezio, A., Restubog, S. L. D., Read, S., Lajom, J. A. L., \& Li, M. (2016). The role of traditionality in the relationships among parental support, career decision-making selfefficacy and career adaptability. Journal of $\mathrm{Vo}$ cational Behavior, 94, 114-123. doi: 10.1016/j. jvb.2016.02.018

Hauck, N., Filho, Machado, W. D. L., Teixeira, M. A. P., \& Bandeira, D. R. (2012). Evidências de validade de marcadores reduzidos para a avaliação da personalidade no modelo dos Cinco Grandes Fatores. Psicologia: Teoria E Pesquisa, 28(4), 417-423. doi: 10.1590/S010237722012000400007

Hauck, N., Filho, Teixeira, M. A. P., Machado, W. L., \& Bandeira, D. R. (2012). Marcadores reduzidos para a avaliação da personalidade em adolescentes. Psico-USF, 17(2), 253-261. doi: $10.1590 / \mathrm{S} 1413-82712012000200009$

Hutz, C. S., \& Bardagi, M. P. (2006). Indecisão profissional, ansiedade e depressão na adolescência: A influência dos estilos parentais. Psico-USF, 11(1), 65-73. doi: 10.1590/S1413- 


\section{8}

John, O. P., Naumann, L. P., \& Soto, C. J. (2008). Paradigm shift to the integrative Big-Five trait taxonomy: History, measurement, and conceptual issues. In O. P. John, R. W. Robins, \& L. A. Pervin (Eds.), Handbook of personality: Theory and research ( $3^{\text {rd }}$ Ed., pp. 114-158). New York: Guilford Press.

Joordan, J. P. (1963). Exploratory behavior: The formation of self and occupational concepts. In D. Super, R. Starisshevsky, R. Matlin, \& J. P. Jordaan (Eds.), Career development: Selfconcept theory (pp. 42-78). New York: College Entrance Board.

Kelly, K. R., \& Lee, W.-C. (2002). Mapping the Domain of Career Decision Problems. Journal of Vocational Behavior, 61(2), 302-326. doi: 10.1006/jvbe. 2001.1858

Lent, R. W., Brown, S. D., \& Hackett, G. (1994). Toward a Unifying Social Cognitive Theory of Career and Academic Interest, Choice, and Performance. Journal of Vocational Behavior, 45(1), 79-122. doi: 10.1006/jvbe.1994.1027

Magalhães, M. O., Alvarenga, P., \& Teixeira, M. A. P. (2012). Relação entre estilos parentais, instabilidade de metas e indecisão vocacional em adolescentes. Revista Brasileira de Orientação Profissional, 13(1), 15-25. Recuperado em http://pepsic.bvsalud.org/pdf/rbop/v13n1/04.pdf

Melo-Silva, L. L., Lassance, M. C. P., \& Soares, D. H. P. (2004). A Orientação Profissional no contexto da Educação e Trabalho. Revista Brasileira de Orientação Profissional, 5(2), 31-52. Recuperado em http://pepsic.bvsalud.org/pdf/rbop/ v5n2/v5n2a05.pdf

Moores, T. T., \& Chang, J. C. J. (2009). Self-efficacy, overconfidence, and the negative effect on subsequent performance: A field study. Information \& Management, 46(2), 69-76. doi: 10.1016/j. im.2008.11.006

Noronha, A. P. P., \& Ambiel, R. A. M. (2008). Estudo Correlacional entre Escala de Aconselhamento Profissional (EAP) e Self-Directed Search (SDS). Interação em Psicologia, 12(1), 21-33. Recuperado em http://ojs.c3sl.ufpr.br/ojs2/index.php/psicologia/article/view/5998/9208

Noronha, A. P. P., Mansão, C. S. M., \& Nunes, M. F. O. (2012). Interesses Profissionais e Personalidade: Análise correlacional a partir do ATPH e BFP. Actualidades em Psicologia, 26(113), 73-
86. Recuperado em http://pepsic.bvsalud.org/ pdf/apsi/v26n113/a06.pdf

Organização para a Cooperação e Desenvolvimento Econômico. (2004). Orientação Escolar e Profissional: Guia para decisores. Lisboa: Gráfica Krispress.

Ourique, L. R., \& Teixeira, M. A. P. (2012). Autoeficácia e personalidade no planejamento de carreira de universitários. Psico-USF, 17(2), 311321. Recuperado em http://www.lume.ufrgs. br/bitstream/handle/10183/98933/000872886. pdf? sequence $=1$

Parslow, R. A., \& Jorm, A. F. (2000). Who uses mental health services in Australia? An analysis of data from the National Survey of Mental Health and Wellbeing. Australian and New Zealand Journal of Psychiatry, 34(6), 997-1008. doi: 10.1046/j.1440-1614.2000.00839.x

Primi, R., Bighetti, C. A., Munhoz, A. H., Noronha, A. P. P., Polydoro, S. A. J., Nucci, E. P. Di, \& Pellegrini, M. C. K. (2002). Personalidade, interesses e habilidades: Um estudo correlacional da BPR-5, LIP e do 16PF. Avaliação Psicológica, 1(1), 61-72. Recuperado em http://pepsic.bvsalud.org/pdf/avp/v1n1/v1n1a07.pdf

Reis, M., Camacho, I., Ramiro, L., Tomé, G., Gomes, P., Gaspar, T., ...Matos, M. G. (2015). A escola e a transição para a universidade: Idades transicionais e o seu impacto na saúde - notas a partir do estudo HBSC/OMS. Journal of Child and Adolescent Psychology, 6(2), 77-92. Recuperado em https://www.researchgate.net/publication $/ 301354028$

Ribeiro, M. A. (2003). Demandas em Orientação Profissional: Um Estudo Exploratório em Escolas Públicas. Revista Brasileira de Orientação Profissional, 4(1-2), 141-151. Recuperado em http://pepsic.bvsalud.org/pdf/rbop/v4n1-2/ v4n1-2a12.pdf

Ribeiro, M. A. (2011). Orientação Profissional: Uma proposta de guia terminológico. In M. A. Ribeiro \& L. L. Melo-Silva (Eds.), Compêndio de Orientação Profissional e de Carreira: Volume 1. Perspectivas históricas e enfoques teóricos clássicos e modernos (pp. 23-66). São Paulo, SP: Vetor.

Ribeiro, M. A., \& Uvaldo, M. da C. C. (2007). Frank Parsons: Trajetória do pioneiro da orientação vocacional, profissional e de carreira. Revista Brasileira de Orientação Profissional, 8(1), 19- 
31. Recuperado em http://pepsic.bvsalud.org/ pdf/rbop/v8n1/v8n1a03.pdf

Rossier, J. (2015). Career Adaptability and Life Designing. In L. Nota \& J. Rossier (Eds.), Handbook of life design: From practice to theory and from theory to practice (pp. 153-167). Göttingen, Germany: Hogrefe.

Saka, N., Gati, I., \& Kelly, K. R. (2008). Emotional and Personality-Related Aspects of Career-DecisionMaking Difficulties. Campbell \& Cellini, 16(4), 403-424. doi: 10.1177/1069072708318900

Santos, M. M., Luna, I. N., \& Bardagi, M. P. (2014). O desafio da orientação profissional com adolescentes no contexto da modernidade líquida. $R e$ vista de Ciências Humanas, 48(2), 263-281. doi: $10.5007 / 2178-4582.2014 v 48 \mathrm{n} 2 \mathrm{p} 263$

Savickas, M. L. (1999). The Psychology of Interests. In M. L. Savickas \& A. R. Spokane (Eds.), $V_{O}$ cational Interests: Meaning, measurement and counseling use (pp. 19-56). Palo Alto, CA: Davies-Black.

Savickas, M. L. (2005). The theory and practice of career construction. In S. D. Brown \& R. W. Lent (Eds.), Career Development and Counseling: Putting Theory and Research to Work (pp. 42-70). Hoboken, NJ: Wiley.

Semensato, A. C., Valeria, C., Bender, C., Camargo, C., Mata, D., Silva, E. D. O., ...Pessini, M. A. (2009). Um estudo qualitativo sobre orientação vocacional e profissional: Direções possíveis, desafios necessários. Akrópolis, 17(1), 29-40. Recuperado em http://revistas.unipar.br/index. php/akropolis/article/view/2840/2108

Silva, A. D., \& Marques, C. (2015). La exploração vocacional em jovens: Estudo com grupos específicos. Revista de Estudios e Investigación en Psicología y Educación, 2(1), 60-66. doi: 10.17979/reipe.2015.2.1.1085

Silva, C. R. E. (2010). Orientação Profissional, mentoring, coaching e counseling: Algumas singularidades e similaridades em práticas. Revista Brasileira de Orientação Profissional, 11(2),
299-309. Recuperado em http://pepsic.bvsalud. org/pdf/rbop/v11n2/v11n2a14.pdf

Teixeira, M. A. P., Bardagi, M. P., \& Hutz, C. S. (2007). Escalas de exploração vocacional (EEV) para universitários. Psicologia em Estudo, 12(1), 195-202. doi: 10.1590/S141373722007000100023

Teixeira, M. A. P., Bardagi, M. P., Lassance, M. C. P., Magalhães, M. O., \& Duarte, M. E. (2012). Career Adapt-Abilities Scale - Brazilian Form: Psychometric properties and relationships to personality. Journal of Vocational Behavior, 80(3), 680-685. doi: 10.1016/j.jvb.2012.01.007

Teixeira, M. A. P., \& Dias, A. C. G. (2011). Escalas de exploração vocacional para estudantes de ensino médio. Estudos de Psicologia (Campinas), 28(1), 89-96. Recuperado em http://www.lume.ufrgs. br/bitstream/handle/10183/98904/000787119. pdf? sequence $=1$

Teixeira, M. A. P., \& Magalhães, M. O. (2001). Escala de indecisão vocacional: Construção de um instrumento para pesquisa. Aletheia, (13), 21-26.

Valentini, F., Teodoro, M. L. M., \& Balbinotti, M. A. A. (2009). Relações entre interesses vocacionais e fatores de personalidade. Revista Brasileira de Orientação Profissional, 10(2), 57-68. Recuperado em http://pepsic.bvsalud.org/pdf/rbop/ v10n2/v10n2a07.pdf

Zoonen, K. V., Kleiboer, A., Beekman, A. T. F., Smit, J. H., Boerema, A. M., \& Cuijpers, P. (2015). Reasons and determinants of helpseeking in people with a subclinical depression. Journal of Affective Disorders, 173, 105-112. doi: 10.1016/j.jad.2014.10.062

Recebido: 12/04/2017

$1^{a}$ revisão: 17/11/2017

Aceite final: 08/12/2017

(c) BY 1 (C) O(s) autor(es), 2018. Acesso aberto. Este artigo está distribuído nos termos da Licença Internacional Creative Commons Atribuição 4.0 (http://creativecommons.org/licenses/by/4.0/), que permite o uso, distribuição e reprodução sem restrições em qualquer meio, desde que você dê crédito apropriado ao(s) autor(es) original(ais) e à fonte, fornecer um link para a licença Creative Commons e indicar se as alterações foram feitas. 